\title{
BOUNDED REAL-ANALYTIC SUBMANIFOLDS OF BANACH SPACES
}

\author{
J. A. JARAMILLO
}

(Communicated by Irwin Kra)

\begin{abstract}
It is shown that, in contrast to the complex case, every paracompact finite-dimensional real-analytic manifold can be embedded as a bounded and closed real-analytic submanifold of every infinite-dimensional real Banach space.
\end{abstract}

In [1], Aurich considered the question of which complex manifolds $X$ can be realized as bounded and closed analytic submanifolds of a complex Banach space $E$. He studied the special case where $X$ is isomorphic to the open unit disk $D$ of $\mathbf{C}$, obtaining that in some cases (when $E$ contains $c_{0}$ or $L^{1} / H_{0}^{1}$ ) $E$ has a bounded and closed analytic submanifold isomorphic to $D$, while in other cases (when $E$ has the analytic Radon-Nikodym property (aRNP)) then there is no bounded and closed analytic submanifold of $E$ isomorphic to $D$. (Recall that $E$ has the aRNP if every bounded holomorphic mapping $\varphi: D \rightarrow E$ admits almost everywhere radial boundary values. See [2] as a reference about this property.)

In this note we are concerned with the analogous question for the real case, and we will see that the situation is quite different from the complex case. Before we state our main result, let us recall that a mapping is said to be proper if the inverse image of each compact subset is compact.

Theorem. If $E$ is an infinite-dimensional real Banach space and $N$ is a natural number, then there exists a map $\psi: \mathbf{R}^{N} \rightarrow E$ such that

(i) $\psi$ is real-analytic, proper and one-to-one,

(ii) $\psi\left(\mathbf{R}^{N}\right)$ is closed and bounded, and

(iii) the differential $(D \psi)(x)$ has rank $N$ at every $x$ in $\mathbf{R}^{N}$.

Proof. Let $U=\left\{\left(z_{1}, \ldots, z_{N}\right) \in \mathbf{C}^{N}:\left|\operatorname{Im}\left(z_{n}\right)\right|<\frac{1}{2}, \forall n=1, \ldots, N\right\}$, and let $E_{\mathrm{C}}$ be the complexification of $E$. Now we consider a normalized basic sequence in $E$ (see e.g. [4, Th. 1.a.5.]), arranged as $\left\{a_{m}^{n}: n=1, \ldots, N ; m \in \mathbf{Z}\right\}$. For

Received by the editors January 20, 1988 and, in revised form, March 14, 1988.

1980 Mathematics Subject Classification (1985 Revision). Primary 32C05, 32K05.

Research partially supported by CAICYT grant $2197 / 83$. 
each $z=\left(z_{1}, \ldots, z_{N}\right) \in U$, each $n=1, \ldots, N$, and each $m \in \mathbf{Z}$, we denote $\varphi_{m}^{n}(z)=\left(1+\left(m-z_{n}\right)^{2}\right)^{-1}$ and then we define the map $\varphi: U \rightarrow E_{\mathbf{C}}$ by

$$
\varphi(z)=\sum_{m \in \mathbf{Z}} \sum_{n=1}^{N} \varphi_{m}^{n}(z) a_{m}^{n}
$$

Note that for each $z=\left(z_{1}, \ldots, z_{N}\right) \in U$, and each $n=1, \ldots, N$, if we select $M_{n} \in \mathbf{N}$ such that $M_{n} \leq\left|\operatorname{Re}\left(z_{n}\right)\right|<M_{n}+1$, we have that

$$
\begin{aligned}
\sum_{m \in \mathbf{Z}} \sum_{n=1}^{N}\left\|\varphi_{m}^{n}(z) a_{m}^{n}\right\| \leq & \sum_{n=1}^{N} \sum_{m \in \mathbf{Z}}\left|\operatorname{Re}\left(1+\left(m-z_{n}\right)^{2}\right)\right|^{-1} \\
\leq & \sum_{n=1}^{N}\left[\sum_{|m| \leq M_{n}}\left(\frac{1}{2}+\left(M_{n}-|m|\right)^{2}\right)^{-1}\right. \\
& \left.+\sum_{|m|>M_{n}}\left(\frac{1}{2}\left(|m|-M_{n}-1\right)^{2}\right)^{-1}\right] \\
& \leq 4\left(2+\pi^{2} / 6\right) N ;
\end{aligned}
$$

therefore the series in the definition of $\varphi$ is absolutely convergent. We consider the restriction $\psi=\left.\varphi\right|_{\mathbf{R}^{N}}$, which takes its values in $E$, and we are going to see that $\psi: \mathbf{R}^{N} \rightarrow E$ has the required properties:

(i) In order to verify that $\psi$ is real-analytic, it is sufficient to show that $\varphi$ is analytic, and this follows from the fact that the series defining $\varphi$ is uniformly convergent on each compact subset of $U$ : indeed, given $R>0$ and $\varepsilon>0$, there exists $M \in \mathbf{N}(M>R)$ such that, for each $z \in U$ with $|\operatorname{Re}(z)| \leq R$,

$$
\left\|\sum_{|m|>M} \sum_{n=1}^{N}\left(1+\left(m-z_{n}\right)^{2}\right)^{-1} a_{m}^{n}\right\| \leq \sum_{|m|>M} \sum_{n=1}^{N}\left(\frac{1}{2}+(|m|-R)^{2}\right)^{-1}<\varepsilon .
$$

Now we prove that $\psi$ is a proper map, that is, the inverse image of each compact subset of $E$ is compact in $\mathbf{R}^{N}$. It is sufficient to show that for every unbounded sequence $\left(x^{j}\right)_{j \in \mathbf{N}} \subset \mathbf{R}^{N}$, the sequence $\left(\psi\left(x^{j}\right)\right)_{j \in \mathbf{N}}$ is not convergent in $E$. Now suppose $\left(x^{j}\right)_{j \in \mathbf{N}}$ is a sequence in $\mathbf{R}^{N}$ such that $\left(\left|x_{n_{0}}^{j}\right|\right)_{j \in \mathbf{N}}$ tends to $+\infty$ for some $n_{0} \in\{1, \ldots, N\}$, but $\left(\psi\left(x^{j}\right)\right)_{j \in \mathbf{N}}$ is convergent, say to $v \in E$. We consider the biorthogonal functionals $\left\{\pi_{m}^{n}: n=1, \ldots, N ; m \in \mathbf{Z}\right\} \subset E^{*}$ associated to the basic sequence $\left\{a_{m}^{n}: n=1, \ldots, N ; m \in \mathbf{Z}\right\}$; there exists $L>0$ such that $\left\|\pi_{m}^{n}\right\| \leq L$, for each $n=1, \ldots, N$ and each $m \in \mathbf{Z}$ (see e.g. [4, 1.b.]). For every $j \in \mathbf{N}$, let $m_{j} \in \mathbf{Z}$ be such that $\left|m_{j}-x_{n_{0}}^{j}\right|<1$; then $\left|\pi_{m_{j}}^{n_{0}}\left(\psi\left(x^{j}\right)-v\right)\right| \leq L\left\|\psi\left(x^{j}\right)-v\right\|$, and both sequences are convergent to 0 . But for each $m \in \mathbf{Z},\left(\left(1+\left(m-x_{n_{0}}^{j}\right)^{2}\right)^{-1}\right)_{j \in \mathbf{N}}=\left(\pi_{m}^{n_{0}}\left(\psi\left(x^{j}\right)\right)\right)_{j \in \mathbf{N}}$ converges to $\pi_{m}^{n_{0}}(v)$, and therefore $\pi_{m}^{n_{0}}(v)=0$; hence for each $j \in \mathbf{N},\left|\pi_{m_{j}}^{n_{0}}\left(\psi\left(x^{j}\right)-v\right)\right|=$ $\pi_{m_{j}}^{n_{0}}\left(\psi\left(x^{j}\right)\right)=\left(1+\left(m_{j}-x_{n_{0}}^{j}\right)^{2}\right)^{-1} \geq \frac{1}{2}$, and this is a contradiction. 
Finally, $\psi$ is one-to-one for, if $x, y \in \mathbf{R}^{N}$ with $x \neq y$, then $x_{n} \neq y_{n}$ for some $n=1, \ldots, N$, and $\psi_{m}^{n}(x)=\psi_{m}^{n}(y)$ for at most one $m \in \mathbf{Z}$.

(ii) Since $\psi$ is proper, it follows that $\psi\left(\mathbf{R}^{N}\right)$ is closed in $E$. On the other hand, $\psi\left(\mathbf{R}^{N}\right)$ is bounded: in fact, as we have seen before, for every $x \in$ $\mathbf{R}^{N}:\|\psi(x)\| \leq 4\left(2+\pi^{2} / 6\right) N$.

(iii) Let $x \in \mathbf{R}^{N}$ be, and let $D \psi(x)$ be the differential of $\psi$ at $x$. We denote the standard basis of $\mathbf{R}^{N}$ by $\left\{e_{1}, \ldots, e_{N}\right\}$, and we are going to see that the vectors $\left\{D \psi(x)\left(e_{1}\right), \ldots, D \psi(x)\left(e_{N}\right)\right\}$ are linearly independent. Since the series defining $\varphi$ is uniformly convergent on compact subsets of $U$, we have that for each $n=1, \ldots, N, D \psi(x)\left(e_{n}\right)=\sum_{m \in \mathbf{Z}} 2\left(m-x_{n}\right)\left(1+\left(m-x_{n}\right)^{2}\right)^{-2} a_{m}^{n}$; therefore if $\sum_{n=1}^{N} \lambda_{n} D \psi(x)\left(e_{n}\right)=0$, then $\lambda_{n}\left(m-x_{n}\right)=0$ for every $m \in \mathbf{Z}$ and every $n=1, \ldots, N$, and hence $\lambda_{n}=0$ for every $n=1, \ldots, N$.

If $X$ is a real-analytic manifold and $E$ is a real Banach space, we say that a real-analytic map $\psi: X \rightarrow E$ is an embedding when $\psi(X)$ is a closed realanalytic submanifold of $E$ and $\psi: X \rightarrow \psi(X)$ is bi-real-analytic.

Corollary. Let $X$ be a paracompact finite-dimensional real-analytic manifold and let $E$ be an infinite-dimensional real Banach space. Then there exists a bounded real-analytic embedding of $X$ into $E$.

Proof. By a classical result due to Grauert [3], there exists a real-analytic embedding of $X$ into $\mathbf{R}^{N}$, for some $N \in \mathbf{N}$, and it follows from the Theorem that there exists a bounded real-analytic embedding of $\mathbf{R}^{N}$ into $E$.

\section{ACKNOWLEDGEMENT}

The author wishes to express his gratitude to Professor R. M. Aron, who suggested the problem, for his help and encouragement.

\section{REFERENCES}

1. V. Aurich, Bounded holomorphic embeddings of the unit disk into Banach spaces, Manuscripta Math. 45 (1983), 61-67.

2. A. Bukhvalov and A. Danilevich, Boundary properties of analytic and harmonic functions with values in Banach spaces, Math. Notes 31 (1982), 104-110.

3. H. Grauert, On Levi's problem and the imbedding of real-analytic manifolds, Ann. of Math. (2) 68 (1958), 460-472.

4. J. Lindenstrauss and L. Tzafriri, Classical Banach spaces. I, Springer-Verlag, Berlin and New York, 1977. SPAIN

Departamento de Análisis Matemático, Universidad Complutense, 28040-Madrid, 